18. Guenther HL, Fuenther HE, Froesch ER, Fleisch H 1982 Effect of insulin-like growth factor on collagen and glycosaminoglycan synthesis by rabbit articular chondrocytes in culture. Experientia 38:979-981

19. Audi L, Carrascosa A, Ballabriga A 1984 Androgen metabolism by human fetal epiphyseal cartilage and its chondrocytes in primary culture. $\mathrm{J}$ Clin Endocrinol Metab 58:819-825

20. Corvol MT, Dumontier MF, Rappaport R 1975 Culture of chondrocytes from the proliferative zone of epiphyseal growth plate cartilage from prepubertal rabbits. Biomedicine 23:103-107
21. Burton K 1956 A study of the conditions and mechanism of the diphenylamine reaction for the colorimetric estimation of DNA. Biochem $J$ 62:315-323

22. Wiebkin OW, Muir H 1977 Synthesis of cartilage-specific proteoglycan by suspension cultures of adult chondrocytes. Biochem J 164:269-272

23. Yasumoto S, Kato Y, Oguri K, Yamagata S, Yamagata T 1980 Maintenance of phenotypic properties by chondrocytes cultured in suspension. Dev Growth Differ 22:445-459

24. Franzén A, Fjörnsson S, Heinegard D 1981 Cartilage proteoglycan aggregate formation. Biochem J 197:669-674

\title{
The Effect of Short-Term Starvation on Mucosal Barrier Function in the Newborn Rabbit*
}

\author{
DEBORAH ROTHMAN, JOHN N. UDALL, KAM Y. PANG, SARA E. KIRKHAM, AND \\ W. ALLAN WALKER \\ Combined Program in Pediatric Gastroenterology and Nutrition, Massachusetts General Hospital and Children's \\ Hospital, Department of Pediatrics, Harvard Medical School, Boston, Massachusetts 02114
}

\begin{abstract}
The compromised human newborn frequently presents with overwhelming feeding problems which lead to inadequate intake. These problems may affect the development of the small intestine, especially mucosal barrier function, leading to increased infections and susceptibility to allergens. To study this, an animal model was established using neonatal rabbits deprived of nutrients from birth until $72 \mathrm{~h}$. Mucosal barrier function was compared in deprived and control (naturally fed 72-hold animals) rabbits by measuring immunoreactive bovine serum albumin in serum $4 \mathrm{~h}$ after intragastric infusion of crystalline bovine serum albumin $(200 \mathrm{mg} / 100 \mathrm{~g}$ body weight). Trypsin activity was measured in rinse fluid obtained from the small intestine. Representative sections of jejunum from control and experimental animals were formalin fixed and stained with hematoxylin and eosin for morphologic comparison. Following the bovine serum albumin feeding, a significantly increased serum immunoreactive bovine serum albumin and significantly decreased trypsin-like activity of the small intestinal rinse fluid was noted in starved animals compared to controls. In addition, the enterocytes of malnourished animals were more cuboidal and contained fewer and smaller supranuclear granules on microscopic examination than the enterocytes of controls. This study suggests that short-term starvation in newborns affects mucosal barrier function. Acute starvation may place newborns at increased risk for infections and allergic disease. (Pediatr Res 19: 727-731, 1985)
\end{abstract}

Received October 9,1984; accepted March 5, 1985

Address correspondence to W. Allan Walker, M.D., Chief, Combined Program in Pediatric Gastroenterology and Nutrition, Massachusetts General Hospital Boston, MA 02114

* Presented in part at the national meeting of the Society for Pediatric Research in Washington, D.C. 1983.

Supported by Grants AM-33506, GM-21700 from the National Institutes of Health. D.R. was a trainee with the Training Grant for Research and Trauma (5T32 GM-07035).
Abbreviations

BSA, bovine serum albumin i-BSA, immunoreactive BSA PBS, phosphate-buffered saline

The compromised human newborn frequently presents with overwhelming feeding problems and grossly inadequate intakes are common. The extent to which this affects the differentiation and development of the small intestine, especially in terms of barrier function, has not been studied. The intestine plays a major role in preventing a variety of intraluminal substances, such as food antigens, microorganisms, and toxins, from penetrating the mucosal barrier and entering the systemic circulation.

There is evidence that under conditions of chronic malnutrition intestinal barrier function is altered in older animals. This observation is based on the finding of increased uptake of both intact and large fragments of protein from the intestinal lumen into the circulation, suggesting that the gut is more permeable under these conditions $(1,2)$.

There is also indirect evidence that a similar phenomenon may occur in malnourished children. Chandra (3) reported an increase in circulating food antibodies in children with severe malnutrition, suggesting that food antigens may cross the gut to induce a systemic antibody response. Similarly, the uptake of pathogens may be increased and this may contribute to the high incidence of gastrointestinal and systemic infections in malnourished children.

Mucosal barrier function in the animal and human studies cited above was compromised only after long periods of nutrient deprivation in the postweaning state. There is no information on how the newborn responds to a similar insult or what effect a short-term period of starvation, which occurs commonly in the clinical setting, would have in the period immediately after birth when the intestine undergoes many changes while adapting to extrauterine life. 
Accordingly, the present study was designed to establish an animal model to examine the effect of short-term malnutrition in the newborn on barrier function. This was assessed by measuring serum BSA concentration following BSA feeding as an indication of intestinal permeability. In addition, small intestinal contents were analyzed for trypsin-like activity to determine whether inadequate intestinal proteolysis was associated with increased macromolecular uptake. Finally, morphological studies of the small intestine were performed to determine if structural differences existed to explain any transport changes noted.

\section{MATERIALS AND METHODS}

Animals. Adult New Zealand White does were raised on Charles River Rabbit food (Country Foods, Syracuse, NY), a plant-based diet devoid of BSA. Does were mated, and on the 31st day of a 31-day gestation, labor was induced by the intramuscular injection of 1.0 USP unit of oxytocin (Sandox Pharmaceuticals, East Hanover, NJ) diluted to $0.2 \mathrm{ml}$ with distilled water.

A total of 46 animals was studied. Each litter of newborn rabbits was divided into two groups matched for weight. A control group was allowed to remain with the mother and suckle ad libitum for $72 \mathrm{~h}$. The experimental group was separated from the mother immediately after birth, placed in an incubator maintained at $30-32^{\circ} \mathrm{C}$ and starved for $72 \mathrm{~h}$. To prevent dehydration the experimental animals were fed water every 4-5 h. A small nipple atttached to a syringe was used to feed these animals. Intake was variable, but $2-10 \mathrm{ml}$ of water were usually consumed at each feeding. Fluid intake was judged to be adequate based on the output of dilute urine.

Transport studies. Lyophilized crystalline bovine serum albumin (Sigma Chemical Co., St. Louis, MO) was suspended in PBS $\left(0.15 \mathrm{M} \mathrm{NaCl}, 7.7 \mathrm{mM} \mathrm{NaH}_{2} \mathrm{PO}_{4} \cdot \mathrm{H}_{2} \mathrm{O}, 16.7 \mathrm{mM} \mathrm{Na} 2 \mathrm{HPO}_{4}, \mathrm{pH}\right.$ 7.4) at a concentration of $100 \mathrm{mg} \mathrm{BSA} / \mathrm{mI}$ PBS. Animals were anesthesized with ether and gavaged with $200 \mathrm{mg} \mathrm{BSA} / 100 \mathrm{~g}$ body weight using a syringe and polyethylene tube (Intramedic, Parsippany, $\mathrm{NJ}$ ).

Newborn rabbit growth data indicate this amount of protein administered by gavage probably represents a physiologic amount of protein. This is based on the observation that newborn rabbits feeding once a day will suckle at least 8 to $10 \mathrm{~g}$ of milk which is $11.0 \%$ protein (4). Therefore, naturally raised infant rabbits will ingest approximately $1.0 \mathrm{~g}$ of protein at a feeding. Because previous work in our laboratory has shown that the maximum concentration of plasma i-BSA after BSA feeding occurred at $4 \mathrm{~h}$, animals were reanesthetized and blood was obtained by cardiac puncture $4 \mathrm{~h}$ after the BSA feeding (4). The blood was allowed to clot and serum was obtained following centrifugation $(1500 \times g)$. Serum i-BSA concentrations were determined on aliquots of samples using electroimmunodiffusion as previously described $(4,5)$.

Small intestinal rinse fluid studies. To assess basal small intestinal rinse trypsin-like activity, selected control $(n=14)$ and starved experimental $(n=14)$ animals were studied. After these animals were anesthetized with ether and cardiac blood was obtained for i-BSA determination, the animals were decapitated. A midline longitudinal incision was then made in the abdomen and a no. 50 polyethylene tube (Intramedic) was inserted into the proximal intestine at the pylorus and tied in place with 3-0 silk suture. The distal ileum was transected proximal to the cecum and the animal placed so that fluid washed through the small intestine would drain from the transected ileum through a funnel into a preweighed vial. Using a syringe and a 22-gauge needle inserted into the no. 50 polyethylene tube in the proximal intestine, $6 \mathrm{ml}$ of cold PBS per $100 \mathrm{~g}$ body weight were flushed through the small intestine. The rinse fluid was drained from the intestine into the preweighed vial with the aid of repeated injections of air. The vial was reweighed to determine the amount of fluid recovered. It was found that $88.6 \% \pm 6.6(\vec{x} \pm S E M)$ of the rinse fluid was recovered $(n=28)$. The fluid was clarified by centrifugation $(1500 \times g)$ and the supernatant stored at $-20^{\circ} \mathrm{C}$ for subsequent testing.

A modification of the Hummel method was used to determine trypsin-like activity in small intestinal rinse fluid (6). In this modification, $2.6 \mathrm{ml}$ of a $0.046 \mathrm{M}$ Tris buffer, containing 0.0115 $\mathrm{M} \mathrm{CaCl}_{2}$ (pH 8.1) was mixed with $0.3 \mathrm{ml}$ of a $0.01 \mathrm{M} \mathrm{p}$ toluenesulfonyl-L-arginine methyl ester (Sigma) solution. To this mixture was added $0.1 \mathrm{ml}$ of undiluted small intestinal rinse fluid. After further mixing, the solution was immediately pipetted into a $3.0 \mathrm{ml}$ quartz cuvette and placed in a model 2400 Gilford automatic recording spectrophotometer (Gilford Instrument Laboratories, Oberlin, $\mathrm{OH}$ ). The rate of hydrolysis of p-toluenesulfonyl-L-arginine methyl ester was determined by the increase in absorbance at $247 \mathrm{~nm}$; enzyme activity was expressed as change in optical density per hour per milliliter fluid. Since the volume of the small intestinal wash used was adjusted for body weight $(6.0 \mathrm{ml} \mathrm{PBS} / 100 \mathrm{~g}$ body weight), proteolytic activity of the rinse fluid was corrected for body weight.

Morphology. Representative sections of jejunum were removed for histologic examination and fixed in $10 \%$ formaldehyde or Karnovsky's fixative. Standard 4- $\mu$ paraffin or $1-\mu$ glycol methacrylate (Sorval Biochemical, Newton, CT) sections were made and stained with hematoxylin and eosin. Sections were coded and examined by a Massachusetts General Hospital staff pathologist (S.E.K.).

Statistics. Statistical analysis was performed using Student's $t$ test.

\section{RESULTS}

Animals. The mean birth weight of 23 control rabbits (suckling) was $53.0 \pm 1.7 \mathrm{~g}(\overrightarrow{\mathrm{x}} \pm \mathrm{SEM})$. After 3 days their mean weight had increased significantly $(p<0.02)$ to $69.5 \pm 3.5 \mathrm{~g}$. The mean birth weight of the 23 experimental animals (starved) was 55.6 $\pm 2.0 \mathrm{~g}$, not statistically different from the controls. However, after 3 days of only water feedings, their mean weight had significantly decreased $(p<0.02)$ to $49.3 \pm 1.8 \mathrm{~g}$ (Fig. 1).

Transport studies. Animals were gavaged with a BSA solution $(200 \mathrm{mg} / \mathrm{ml}$ PBS$)$ given in a dose proportional to body weight $(200 \mathrm{mg} / 100 \mathrm{~g}$ body weight). The presence of i-BSA in the plasma was measured $4 \mathrm{~h}$ later by electroimmunodiffusion. The

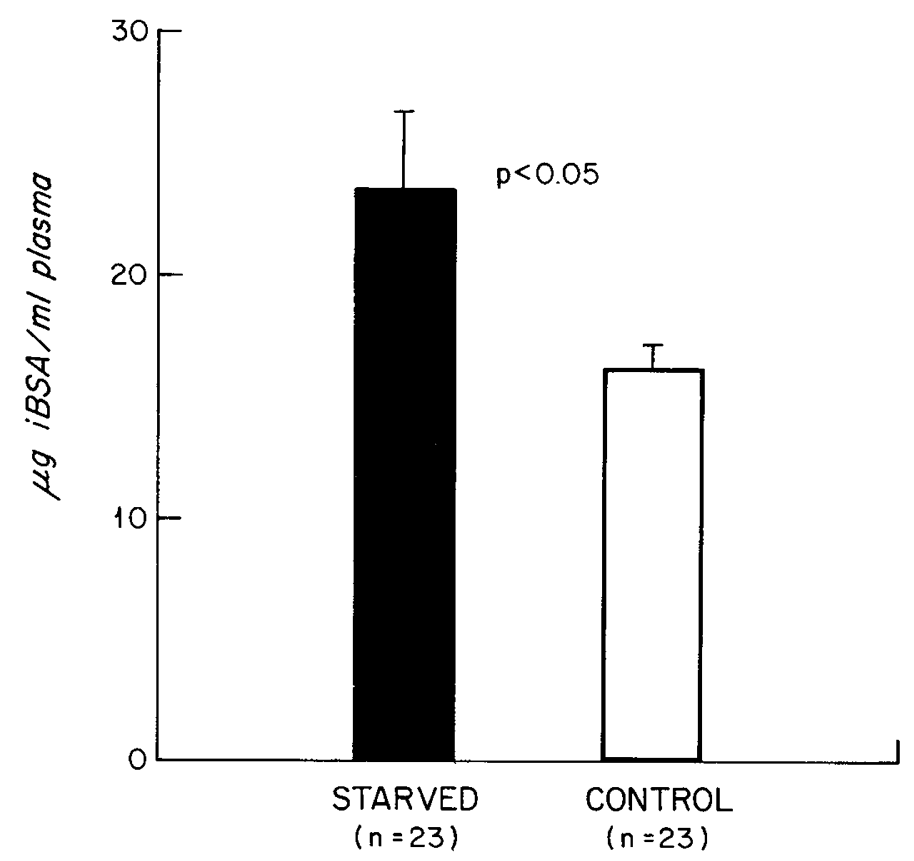

Fig. 1. Immunoreactive BSA $(\mu \mathrm{g} / \mathrm{ml})$ in plasma of starved or fed rabbits $4 \mathrm{~h}$ after gavage feeding of $200 \mathrm{mg}$ BSA per $100 \mathrm{~g}$ body weight. 
starved animals had $23.5 \pm 3.0 \mu \mathrm{g}$ i-BSA per milliliter plasma $(\overrightarrow{\mathrm{x}}$ \pm SEM) and the fed animals had $16.2 \pm 1.1 \mu \mathrm{g}$ i-BSA per milliliter plasma $(p<0.05)$ (Fig. 2).

Small intestinal rinse fluid studies. Trypsin-like activity of the small intestinal rinse fluid was measured by a modification of the Hummel method (6). Mean basal trypsin-like activity in the starved group was $3.5 \pm 0.6$ change in optical density per hour

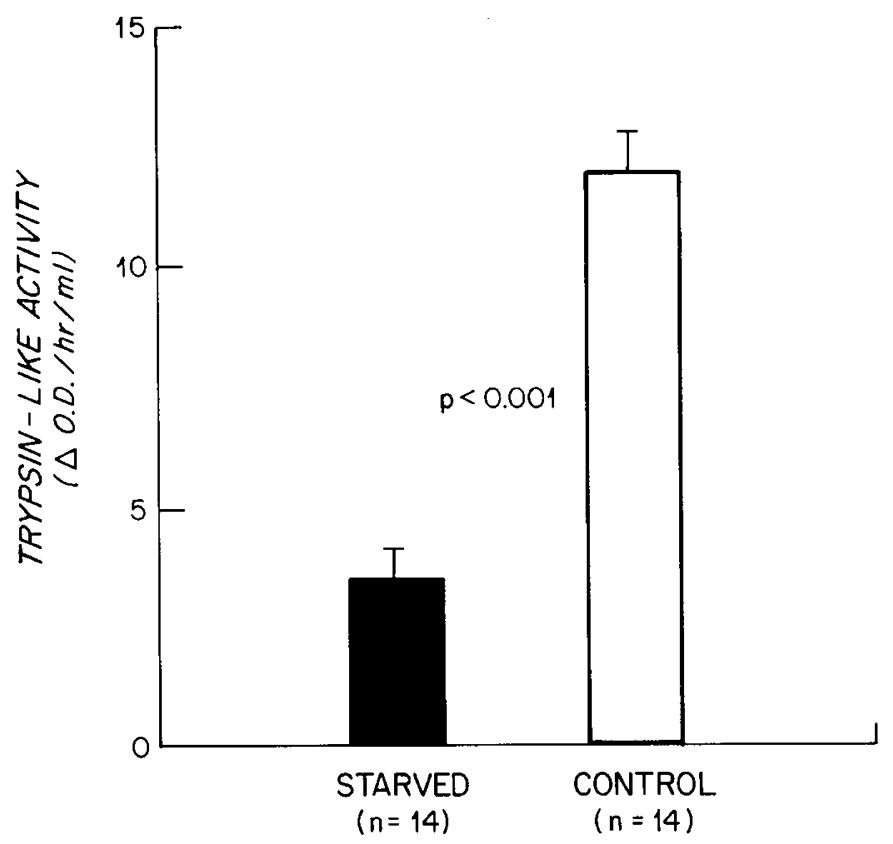

Fig. 2. Trypsin-like activity ( $\Delta$ optical density $/ \mathrm{h} / \mathrm{ml}$ ) in small intestinal rinse fluid obtained from animals or fed for the first $72 \mathrm{~h}$ of life. per milliliter versus $10.9 \pm 1.3$ change in optical density per hour per milliliter for the controls $(p<0.001)$.

Morphology. Changes in mucosal morphology were noted when sections from starved animals (Fig. 3) were compared with controls (Fig. 4). The jejunal villi of the starved newborns appeared more widely spaced, shorter, and more irregular in shape than those of the controls. Enterocytes of the acutely malnourished animals were cuboidal and contained fewer and smaller supranuclear vacuoles than controls. Although goblet cells were present, they were less conspicuous in the starved animals than in the controls.

\section{DISCUSSION}

Following BSA feeding, a significantly increased quantity of iBSA was recovered from the plasma of animals starved for only 3 days compared to fed controls. This observation suggests that intestinal mucosal barrier function may be disrupted early in starvation in the newborn.

There are several factors that may be responsible for this. First, pancreatic function may be compromised. Both animal and human studies have reported pancreatic insufficiency in chronic malnutrition in the postweaning state $(7,8)$. Our observation that small intestinal rinse fluid from the newborn animals starved for 3 days was decreased in trypsin-like activity suggests that the pancreas may be sensitive to a brief nutritional insult as well. The effect of short-term starvation on the pancreas has been studied by Wachstein and Meisel (9). They showed that rats kept on a protein-free diet showed morphologic changes of the pancreas after 10-12 days (9). An alternative explanation is that the decreased luminal trypsin-like activity may be the result of the lack of stimulation of food and not pancreatic insufficiency. Regardless of the mechanisms contributing to decreased luminal trypsin-like activity, a decreased intestinal proteolytic activity

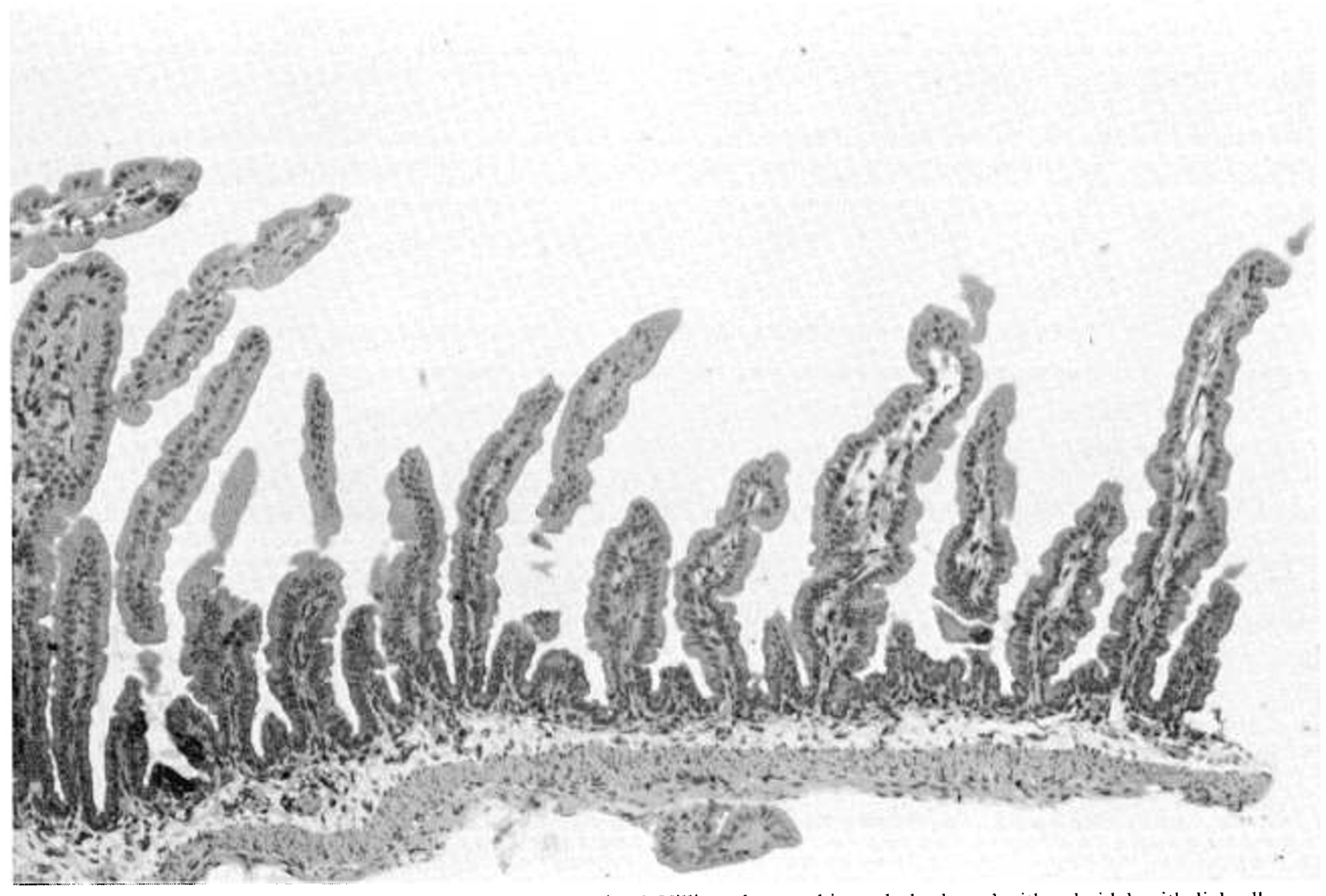

Fig. 3. Representative section of jejunum from a starved animal. Villiare short and irregularly shaped with cuboidal epithelial cells. 


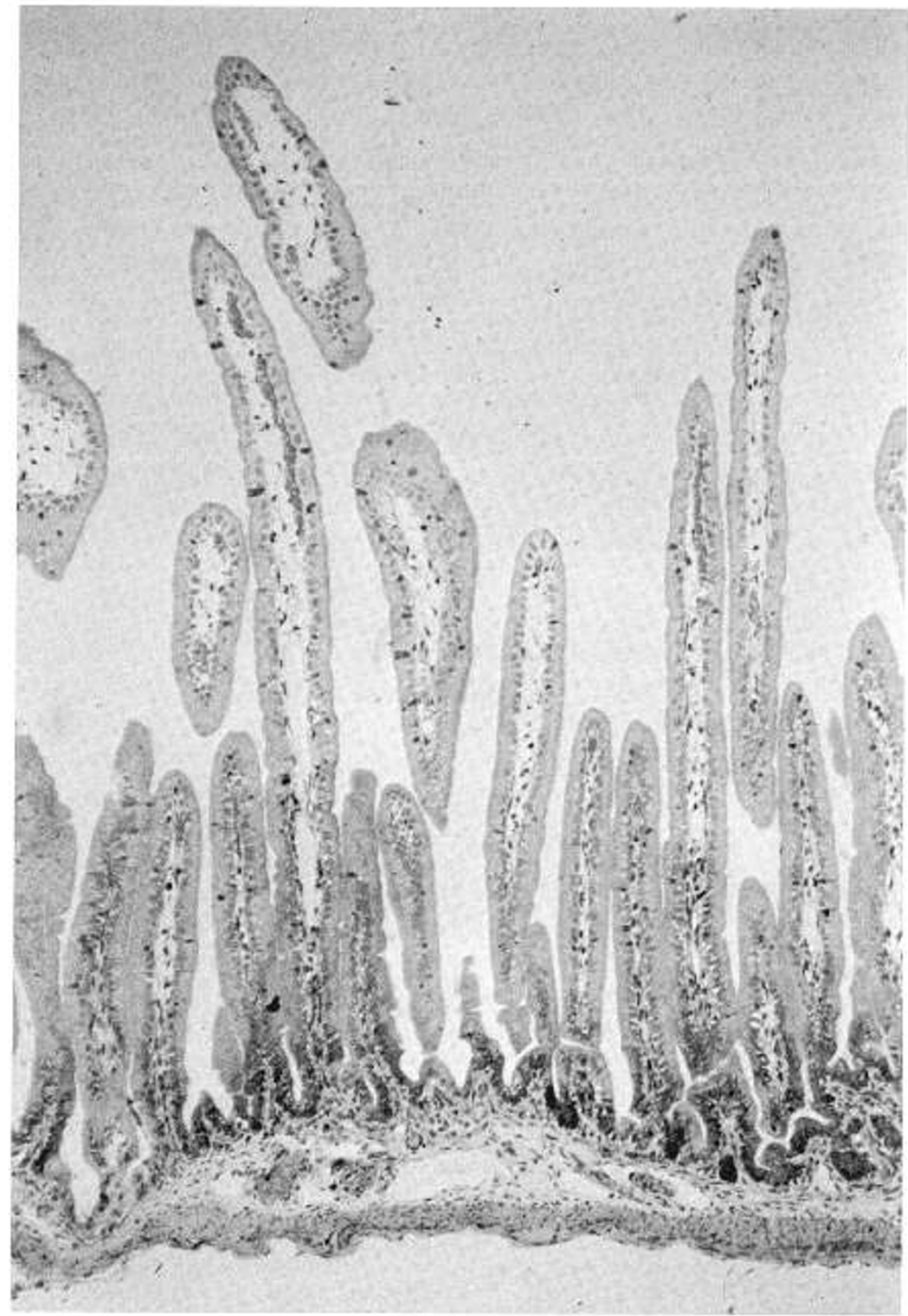

Fig. 4. Representative section of jejunum from a control animal. Villi are long and finger-like with columnar epithelial cells containing prominent supranuclear vacuoles.

may be associated with incomplete digestion of exogenous protein with a concomitant increase in the intraluminal concentration and uptake of BSA. Previous work in our laboratory has shown intestinal uptake of macromolecules correlates with the intestinal concentrations of the substance (1). We have also demonstrated that feeding trypsin inhibitors decreases small intestinal rinse fluid trypsin-like activity and this is associated with increased amounts of plasma i-BSA following a BSA feed (10). This supports the idea that proteolytic activity within the intestinal lumen may act as a regulator of the transmission of exogenous proteins from the gut to the plasma (11).

Second, chronic nutrient deprivation is known to cause atrophy of the small intestine and disruption of mucosal integrity. We noted early microscopic changes in the small intestine of acutely malnourished animals. Therefore we assume that the mucosal barrier to macromolecules may be affected.

Third, not only was the experimental group deprived of calories, protein, and fat, but also of all the hormones in breast milk, most notably epidermal growth factor, known to have a trophic effect on the small intestine (12). Breast milk compared to formula or water may enhance intestinal host defense. In earlier studies we have noted that breast-fed rabbits have significantly less i-BSA in their plasma than formula-fed animals following a BSA feeding (13).

Increased pinocytotic activity of intestinal epithelial cells, alterations in gastric and/or intestinal $\mathrm{pH}$, changes in the glycocalyx or mucous secretion, and decreased quantities of enterocyte lysosomal enzymes may also be factors operating in the starved animals to enhance the uptake of intact proteins (14). Studies to examine contributions by these factors are in progress.

Our data suggest that the newborn with an incompletely developed gastrointestinal tract is susceptible to increased uptake of potential antigens and toxins following short-term nutrient deprivation. Although it has been known for some time that chronic malnutrition of at least one month's duration can alter intestinal barrier function, this is the first report in which a brief 
period of nutritional deprivation has been shown to have an objective effect on the small intestine.

\section{REFERENCES}

1. Rothman D, Latham MC, Walker WA 1982 Transport of macromolecules in malnourished animals. I. Evidence of increased uptake of intestinal antigens. Nutr Res 2:467-473

2. Worthington DB, Boatmann ES, Kenny GE 1974 Intestinal absorption of intact protein in normal and protein-deficient rats. Am J Clin Nutr 27:276286

3. Chandra RK 1975 Food antibodies in malnutrition. Arch Dis Child 50:532534

4. Udall JN, Pang K, Fritze L, Kleinman R, Walker WA 1981 Development of gastrointestinal mucosal barrier. I. The effect of age on intestinal permeability to macromolecules. Pediatr Res 15:241-244

5. Laurell CB 1966 Quantitative estimation of proteins by electrophoresis in agarose gel containing antibodies. Anal Biochem 15:45-52

6. Hummel BC 1959 A modified spectrophotometric determination of chymotrypsin, trypsin and thrombin. Can J Biochem 37:1393-1399
7. Barbezat MS, Hausen JDL 1968 The exocrine pancreas and protein-calorie malnutrition. Pediatrics 42:77-92

8. Hatch T, Branski D, Krasner J, Lebenthal E 1978 The effect of acquired postnatal mainutrition on pancreatic enzymes in the rat. Pediatr Res 12:435

9. Wachstein M, Meisel E 1954 Relation of dietary protein levels to pancreatic damage in the rat. Proc Soc Exp Med 85:314-317

10. Udall JN, Bloch J, Vachino G, Feldman P, Walker WA 1984 Development of the gastrointestinal mucosal barrier. IV. The effect of inhibition of proteolysis on the uptake of macromolecules by the intestine. Biol Neonate 45:289-295

11. Telemo E, Westrom BR, Karlsson BW 1982 Proteolytic activity as a regulator of the transmission of orally fed proteins from the gut to the blood serum in the suckling rat. Biol Neonate 41:85-93

12. Carpenter G 1980 Epidermal growth factor is a major growth-promoting agent in human milk. Science 210:198-199

13. Udall JN, Colony P, Fritze L, Pang K, Trier JS, Walker WA 1981 Development of gastrointestinal mucosal barrier. II. The effect of natural versus artificial feeding on intestinal permeability to macromolecules. Pediatr Res 15:245249

14. Udall JN, Walker WA 1982 The physiologic and pathologic basis for the transport of macromolecules across the intestinal tract. J Pediatr Gastroenterol Nutr 1:295-301

\title{
Pulmonary Toxicity of Monocrotaline Differs at Critical Periods of Lung Development
}

\author{
LIVIA TODD, MICHELLE MULLEN, PETER M. OLLEY, AND MARLENE RABINOVITCH \\ Departments of Cardiology and Pathology, Research Institute. The Hospital for Sick Children and Departments \\ of Paediatrics and Pathology, University of Toronto, Toronto, Canada
}

\begin{abstract}
We injected 3-day-old (neonatal), 8-day-old (infant), and 8-wk-old (adult) Sprague-Dawley rats with monocrotaline to examine the effect of a toxic agent at various stages of lung development. Two and four weeks after injection the rats were killed and the heart and lungs removed: the right and left ventricles were separated and weighed, the pulmonary artery was injected with bariumgelatin, and the lung was fixed in the inflated state. Morphometric techniques were applied to assess lung volume, alveolar size and number, and arterial size, muscularity, and concentration relative to alveolar. Rats injected with monocrotaline in the neonatal period did not survive to 3 wk. After 2 wk, there was no significant right ventricular hypertrophy and pulmonary vascular changes were no worse than in the other rat groups injected with monocrotaline, but alveolar development was severely impaired; less than one-third the normal number was present. Rats injected with monocrotaline in infancy had normal alveolar development. After 2 -wk, the arterial changes, i.e. extension of muscle into peripheral arteries, medial hypertrophy of muscular arteries, and decreased arterial concentration relative to alveolar were similar to those observed in adult rats. After 4 wk, there was a decrease in medial hypertro-
\end{abstract}

Received September 28, 1984; accepted March 11, 1985.

Requests for reprints Dr. Marlene Rabinovitch, Department of Cardiology, The Hospital for Sick Children, 555 University Avenue, Toronto, Ontario, Canada M5G 1 X8.

Supported by the Department of Anatomy and the Independent Studies Program at Trinity College, University of Toronto and by the Ontario Heart Foundation (Grant 0HF4-14). M.R. is a Research Associate of the Ontario Heart Foundation. phy associated with growth in artery size and only a lack of regression of right ventricular weight. In adult rats, after 4 wk medial hypertrophy became progressively more severe, the arterial concentration relative to alveolar decreased further, and right ventricular hypertrophy developed. Thus, exposure to a toxic agent in the newborn period may have a critical effect on alveolar development, whereas arterial changes induced by a toxic agent during infancy may regress if there is potential for growth of the pulmonary vascular bed.

A toxic insult may be expected to affect lung development. The newborn rat exposed chronically to hyperoxia will have impaired alveolar and arterial growth $(1,2)$ and the young rat exposed chronically to hypoxia will have more severe pulmonary vascular changes than the adult (3). Monocrotaline is a cytotoxic compound derived from the seeds of Crotalaria spectabilis (4). When administered orally or by injection in adult rats, the pyrrolizidine alkaloid metabolite induces endothelial injury and causes progressive pulmonary vascular changes and right ventricular hypertrophy (5-9). The effects it might have in the developing lung have not been previously studied and could contribute to understanding the response to inflammation and mechanisms of pulmonary hypertension in the newborn and young infant. Meyrick et al. (10) observed that the developing lung passes through two critical periods of growth, one related to alveolar multiplication (3-8 days of age) the other to arterial 